\title{
Accumulation of somatic mutation in mitochondrial DNA extracted from peripheral blood cells in diabetic patients
}

\author{
T. Nomiyama ${ }^{1}$, Y. Tanaka ${ }^{1}$, N. Hattori ${ }^{2}$, K. Nishimaki ${ }^{3}$, K. Nagasaka ${ }^{3}$, R. Kawamori ${ }^{1}$, S. Ohta ${ }^{3}$ \\ ${ }^{1}$ Department of Medicine, Metabolism and Endocrinology, ${ }^{2}$ Department of Neurology, School of Medicine, Juntendo University, \\ Tokyo, Japan \\ ${ }^{3}$ Department of Biochemistry and Cell Biology, Institute of Gerontology, Nippon Medical School, Kawasaki-city, Japan
}

\begin{abstract}
Aims/hypothesis. A point mutation of mitochondrial DNA at nucleotide number $3243 A$ to $G$ is responsible for both the major genetic aetiologies of the MELAS (mitochondrial myopathy, encephalopathy, lactic acidosis and stroke-like episodes) and mitochondrial diabetes. Otherwise, this mutation is also reported to occur as an acquired somatic mutation, possibly due to oxidative stress. Since diabetes can cause severe oxidative stress, we hypothesize that the accumulation of the somatic $3243 A$ to $G$ mutation in mitochondrial DNA can be accelerated by diabetes.

Methods. DNA was extracted from blood samples of 290 non-diabetic healthy subjects (age 20-60) including 98 newborn infants and from 383 patients with Type II (non-insulin-dependent) diabetes mellitus (age 18-80). The extent of somatic $3243 A$ to $G$ mutation to total mitochondrial DNA was detected by real-time PCR using the TaqMan Probe.
\end{abstract}

Results. Whereas the level of the $3243 A$ to $G$ mutation was negligible in the newborn group, it was increased in healthy subjects who were 20 to 29 and 41 to 60 years of age, suggesting that this mutation was somatic. In the diabetic patients the mutation rate increased along with age and the duration of diabetes. In the middle-aged group (age 41-60), the $3243 A$ to $G$ mutation accumulates fourfold higher in the diabetic patients than the healthy subjects. Moreover, multiple regression analysis showed that the most critical factor associated with this mutation in diabetic patients was the duration of diabetes.

Conclusion/interpretation. Diabetes accelerates the accumulation of the somatic $3243 A$ to $G$ mutation in mitochondrial DNA, which can accelerate the ageing process. This somatic mutation could possibly be a new marker for estimating the duration of diabetes. [Diabetologia (2002) 45:1577-1583]

Keywords Mitochondrial DNA, somatic mutation, oxidative stress, age acceleration, long-term marker for diabetic duration.
Received: 31 December 2001 / Revised: 10 April 2002

Published online: 11 July 2002

(C) Springer-Verlag 2002

Corresponding author: Dr. Y. Tanaka, Department of Medicine, Metabolism and Endocrinology, School of Medicine, Juntendo University, 2-1-1, Hongo, Bunkyo-ku, Tokyo, 113-8421 Japan. E-mail: y-tanaka@med.juntendo.ac.jp

Abbreviations: DNA, Deoxyribonucleic acid; mtDNA, mitochondrial DNA; ROS, reactive oxygen species; A3243G, 3243 A to $\mathrm{G}$ mutation; nDNA, nuclear DNA; MELAS, mitochondrial myopathy, encephalopathy, lactic acidosis and stroke-like episodes; PCR, polymerase chain reaction; ATP, adenosine $5^{\prime}$-triphosphate; dATP, deoxy adenosine $5^{\prime}$-triphosphate; dGTP, deoxy guanosine $5^{\prime}$-triphosphate; dCTP, deoxy cytosine $5^{\prime}$-triphophate; dTTP, deoxy timine $5^{\prime}$-triphosphate.
A point mutation of mtDNA at nucleotide point 3243 $A$ to $G(A 3243 G)$ is a major genetic cause of diabetes [1]. In Japan, approximately $1 \%$ of diabetic patients were estimated to have this mutation in blood cells [2]. This point mutation was at first identified as the gene responsible for the MELAS subgroup of mitochondrial encephalomyopathies $[3,4,5]$. On the other hand, it has also been reported that point mutations and deletions of mtDNA accumulate dependent on age in healthy subjects possibly due to sustained exposure to ROS during life in non-dividing cells $[6,7]$. These acquired mutations are called somatic mutations, and recognized as a marker and a cause of ageing [8]. mtDNA has a 5- to 20-fold higher somatic mutation 
rate compared with nDNA, because (i) mtDNA is located near the respiratory chain, potentially generating ROS; (ii) mitochondria have less efficient DNA repair systems compared with the nucleus; (iii) mtDNA is not protected by histone like nDNA; (iv) mtDNA polymerase-gamma has a relatively high insertion error-rate of about 1 per 7000 bases; (v) the single stranded D-loop replication mechanism of mtDNA favours the intermediate formation of clover leaf structures at the $t R N A$ genes; (vi) mutation in a single mtDNA molecule can be propagated within cells due to the continuous turnover of mtDNA in all cells of an organism [9]. The mtDNA 3243 nucleotide position is one of the vulnerable spots for somatic mutation, because this point has remained single stranded for a relatively long time during the duplication of mtDNA. Furthermore, the damage of mtDNA by ROS could result in errors in the synthesis of respiratory chain proteins, leading to dysfunction in ATP production and increasing a leak of ROS to cause further mtDNA mutations. This vicious cycle can decrease cell viability and cause the ageing process.

Somatic mtDNA mutations not only accumulate during normal ageing [10] but also in various diseases associated with the enhancement of ROS, for example, cancer [11], coronary atherosclerotic heart disease [12] and neurodegenerative diseases [13, 14, 15]. In diabetes, both hyperglycaemia and hyperinsulinaemia can increase oxidative stress. While hyperglycaemia produces ROS through the polyol pathway [16], glyco-oxidation [17], and impairment of superoxide dismutase by glycation [18], chronic hyperinsulinaemia also increases ROS production through the activation of endothelial NAD(P)H oxidase [19]. Accordingly, diabetes has been suggested to enhance DNA damage by ROS [20], which, in turn, can cause diabetic complications [21]. We hypothesized that the accumulation of the somatic mtDNA $A 3243 G$ could be accelerated by diabetes. Although blood cells are not expected to accumulate mutant $m t D N A$ because of their rapid turn over, DNA samples are easily collected from blood for statistical analysis. Therefore, it is essential to develop a method to detect small amounts of mutant $m t D N A$, co-existing with wild-type $m t D N A$. We developed a method to measure the mtDNA $A 3243 G$ at a level of less than $0.1 \%$ in mtDNA using TaqMan Probe. Then, we evaluated somatic $m t D N A$ $A 3243 G$ in diabetic patients and non-diabetic healthy subjects.

\section{Subjects and methods}

Patients and control subjects. Total DNA was prepared from peripheral blood cells using the QIAamp DNA Blood Mini Kit (Qiagen, Calif., USA) and stored at $-20^{\circ} \mathrm{C}$. A total of 383 Japanese patients with Type II (non-insulin-dependent) diabetes mellitus patients diagnosed by the diabetes criteria of the Japan Diabetes Society (247 men and 136 women, 18-80 years-old, mean \pm SEM; $58.87 \pm 0.59$ years) were recruited from the outpatient clinic of Juntendo University Hospital (Tokyo, Japan). Patients with a diagnosis of MELAS or mitochondrial diabetes were not included in the study group. All patients gave their written informed consent before they enrolled in the study, which was approved by the Ethics Committee of Juntendo University. Blood from the umbilical vein of neonates was obtained with the written informed consent from their parents at the second hospital of Nippon Medical School (Kawasaki, Japan). Healthy adult-volunteers were also recruited after giving their informed consent. Both the healthy subjects and the diabetic patients were divided into three age groups.

Quantitative detection of mtDNA with a A3243G point mutation. For the detection of a small amount of $m t D N A$ at nucleotide position 3243, the amplification refractory-mutationsystem method was applied [15]. A PCR primer with a sequence matched to the mutated $m t D N A$ at the $3^{\prime}$-end was used for selective amplification of the mutated $m t D N A$. The PCR products were quantified with the TaqMan probe using an automatic sequence detection system (Prism 7700 system; Applied Biosystems) at each step of amplification. The primers used were ATTAAAGTCCTACGTGATC (3048-3066) and ATGCGATTACCGGGCC (3258-3243) for detecting the mutant $m t D N A$, while GCCTTCCCCCGTAAATGATAT (31633183) and GAAGAGGAATTGAACCTCTGACTG (3298$3275)$ were used for quantifying total mtDNA. The nucleotide sequence of the TaqMan probe was TGCCATCTTAACAA ACCCTGTTCTTGGGTT (3241-3213). The oligonucleotides were highly purified by high performance liquid chromatography (HPLC) and repeat freezing and thawing was avoided to not contaminate partially hydrolysed primers. PCR was repeated for 40 cycles at $95^{\circ} \mathrm{C}$ for $15 \mathrm{~s}, 51^{\circ} \mathrm{C}$ for $10 \mathrm{~s}$ and $57^{\circ} \mathrm{C}$ for $1 \mathrm{~min}$. The reaction mixture $(25 \mu \mathrm{l})$ contained ABI TaqMan buffer A, plus $0.05 \%$ glycerol, $2.5 \mathrm{mmol} / \mathrm{l} \mathrm{MgCl}_{2}$, $0.16 \mathrm{mmol} / \mathrm{l}$ each of dATP, dGTP, dCTP and dTTP, 0.6 units of Ampli Taq Gold (ABI), $100 \mathrm{nmol} / \mathrm{l}$ TaqMan probe and $200 \mathrm{nmol} / \mathrm{l}$ of each of the forward and reverse primers for quantifying total mtDNA, or $300 \mathrm{nmol} / \mathrm{l}$ of the forward primer and $100 \mathrm{nmol} / \mathrm{l}$ of the reverse primer for quantifying mutant $m t D N A$.

Standard curves for quantifying total and mutant $m t D N A$ were obtained using plasmids with the wild-type or the mutant $m t D N A$ fragments respectively. The correlation coefficient in each experiment was obtained in the range of the standard samples from $10^{3}$ to $10^{-1} \mathrm{fg}$. When the correlation coefficient was lower than 0.99 , the experiment was reported to ensure intra and inter reproducibility of each assay. The mtDNA fragments with or without mutation were cloned from a MELAS patient and a healthy control subject, respectively. The total mtDNA concentration was initially measured in each sample as described above and the concentration was adjusted just before examining the mutant mtDNA. All samples were randomly rearranged to avoid any bias. PCR measurements were done five times for each sample in different PCR-wells and the average of the three medium values out of the five measurements was calculated to eliminate false values. By taking three mean values out of five measurements, the average standard deviation could be restricted to within $50 \%$. The total mtDNA content was examined again at the same time as the mutant $m t D N A$ content and a ratio was calculated.

Statistical analysis. Data are expressed as the means \pm SEM. The statistical significance of the differences in mean values and frequencies was evaluated by Student's $t$ test or Scheffe's multiple comparison test. To assess the relation of parameters 
A

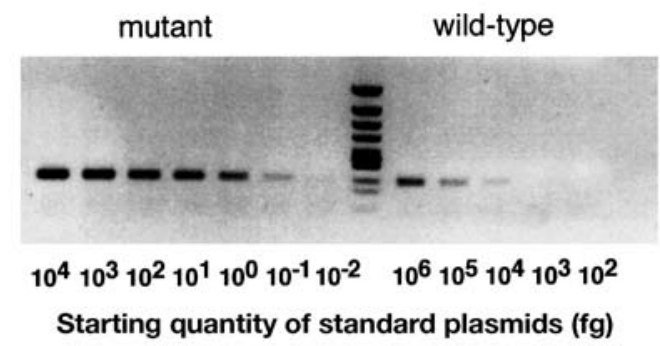

B

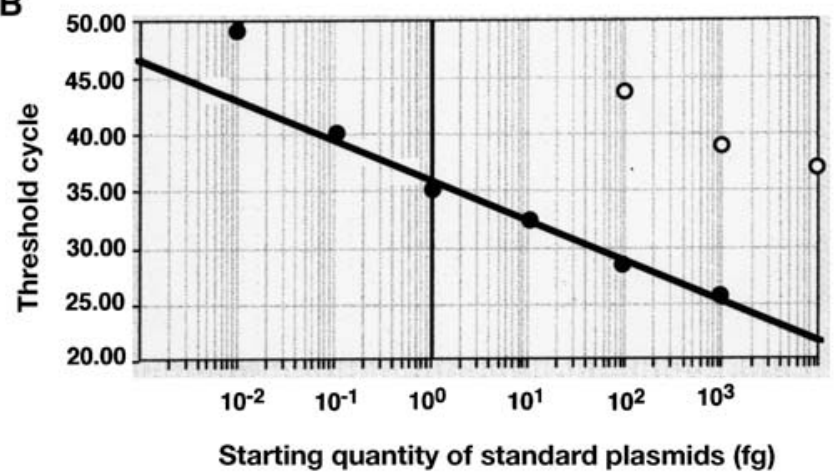

Fig. 1A, B. A method for quantifying a small amount of $A 3243 G$ mutant $m t D N A$. A PCR was done for 35 cycles using primers for the mutant $m t D N A$ (Left) or total mtDNA (Right) using the indicated quantity of standard plasmids (left, $A 3243 G$ mutant; right, wild-type) as a template under the conditions described in Subjects and methods. PCR products were subjected to agarose gel electrophoresis, followed by ethidium bromide staining. B A standard curve for quantifying the amount for mutant $m t D N A$. The PCR products were monitored by fluorescence intensity from the TaqMan probe at each PCR cycle. PCR was done as described in Subjects and methods using the standard plasmids with (closed circles) or without (open circles) the $A 3243 G$ mutant

related to diabetes and the somatic $m t D N A A 3243 G$, multiple regression analysis was carried out. Then the stepwise forward selection method was applied for a more detailed analysis. Differences were considered statistically significant at a $p$ value of less than 0.05 .

\section{Results}

Development of a method for quantifying a small amount of $A 3243 G$ mutation in mtDNA. Since only a minor accumulation of the mutant $m t D N A$ was expected in blood cells, we developed a method for quantifying minute amounts of mutant mtDNA. Under the strict PCR conditions described in Subjects and meth$o d s$, only mutant $m t D N A$ with $n p 3243$ was amplified, while a 100,000-fold excess of wild-type mtDNA was not detected by agarose electrophoresis (Fig. 1A). The amplified DNA fragment was confirmed to be the corresponding mtDNA region by sequencing. In addition, no band was seen when total DNA derived from HeLa cells lacking mtDNA (so-called $\rho^{0}$ cells) was used as a template. This finding excluded any influence of nuclear pseudogenes of the $m t D N A$ fragments. For quantitative analysis, the amplified DNA fragment was monitored with the TaqMan probe in DNA sequence detection system 7700 and estimated using plasmids containing mtDNA fragments with or without the $A 3243 G$ mutation as standard. Nuclear DNA from $\rho^{0}$ cells did not disturb the measurement of mutant $m t D N A$ in a mixture of the nuclear DNA and the standard plasmid. Figure 1B shows a wide range of linearity for quantification of the mutant $m t D N A$, while even a 10,000-fold excess of wild-type $m t D N A$ could not be detected. In addition, since all the samples were randomly rearranged and measurement was repeated five times, any bias could be avoided to confirm the reliability of this experiment. In conclusion, the mutant $m t D N A$ from $0.001 \%$ to $0.1 \%$ of total mtDNA could be quantified by this method.

The Rate of $A 3243 G$ mutant mtDNA. The characteristics of the subjects are shown in Table 1. The stable fractions of glycohaemoglobin $\left(\mathrm{HbA}_{1 \mathrm{c}}\right.$; normal range is under $5.8 \%$ ), a marker of glycaemic control for a few months, was lower in the elderly group (61-80 years old) than in the middle-aged group $(p<0.05)$ and

Table 1. Lists of non-diabetic healthy subjects and diabetic patients

\section{Healthy subjects}

age (years) $\quad n$

$\begin{array}{cc}0 & 98 \\ 20-29 & 93 \\ 41-60 & 99\end{array}$

Diabetic patients

\begin{tabular}{lccccc} 
Age (years) & $n$ & Male/female & Duration of diabetes (years) & $\mathrm{HbA}_{1 \mathrm{c}}(\%)$ & $\mathrm{BMI}\left(\mathrm{kg} / \mathrm{m}^{2}\right)$ \\
\hline $18-40$ & 27 & $15 / 11$ & $7.4 \pm 1.35$ & $7.3 \pm 0.26$ & $26.6 \pm 1.16$ \\
$41-60$ & 174 & $114 / 59$ & $10.8 \pm 0.60^{\mathrm{a}}$ & $7.3 \pm 0.11$ & $23.0 \pm 0.24^{\mathrm{a}}$ \\
$61-80$ & 188 & $118 / 66$ & $14.5 \pm 0.75^{\mathrm{a}, \mathrm{c}}$ & $7.0 \pm 0.11^{\mathrm{b}}$ & $23.0 \pm 0.25^{\mathrm{a}}$ \\
\hline
\end{tabular}

${ }^{\text {a }} p<0.01$ compared with young group

${ }^{\mathrm{b}} p<0.05$ compared with middle-aged group
${ }^{\mathrm{c}} p<0.01$ compared with middle-aged group

$H b A_{1 c}$ stable fraction of glycohaemoglobin 


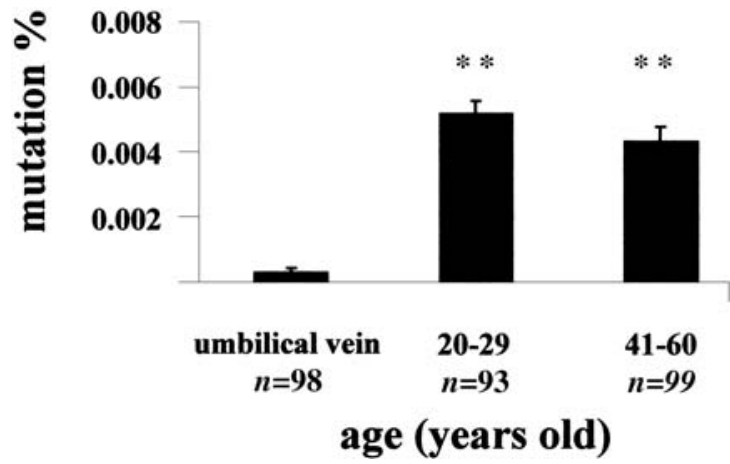

Fig. 2. Ratio of $A 3243 G$ mutant $m t D N A$ to total mtDNA in non-diabetic subjects. The ratio of $A 3243 G$ mutant $m t D N A$ to wild-type $m t D N A$ was examined as described in Subjects and methods. mutation \%; percentage of total mtDNA mutated. $* * p<0.01$ compared with the umbilical vein samples

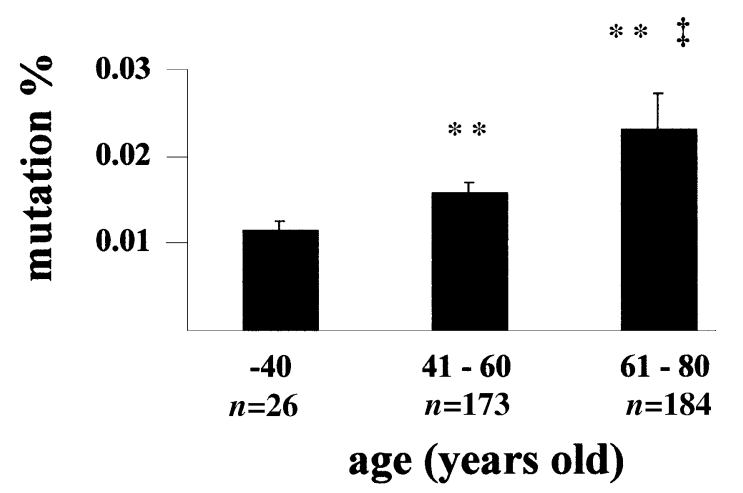

Fig. 3. Ratio of A3243G mutant mtDNA to total mtDNA in age-divided diabetic patients. $* * p<0.01$ compared with the young group. $\ddagger p<0.05$ compared with the middle-aged group

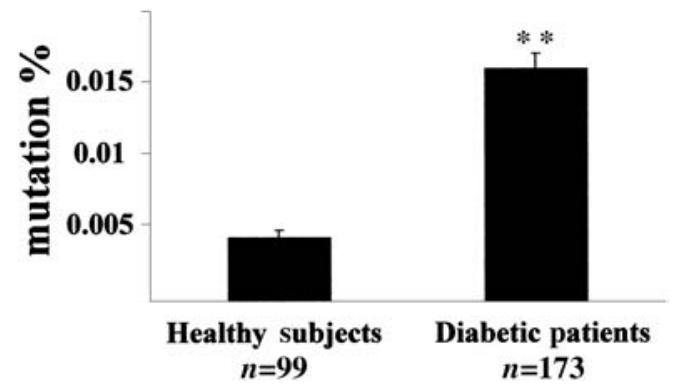

Fig. 4. Comparison of mtDNA A3243G in 41-60 year-old diabetic patients and non-diabetic subjects. $* * p<0.01$ compared with the age-matched non-diabetic subjects

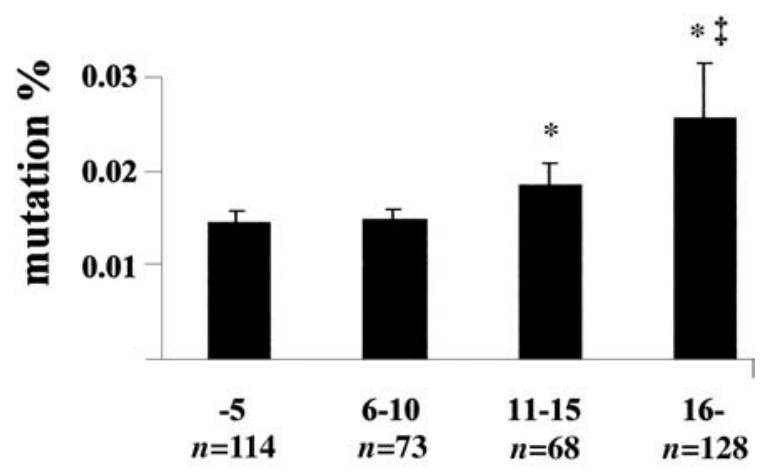

duration(years)

Fig. 5. mtDNA A3243G in duration-divided diabetic patients. $* p<0.05$ compared with $1-5$ years, $\ddagger p<0.05$ compared with 6-10 years

Table 2. Analysis of clinical factors associated with mtDNA A3243G in diabetic patients with multiple regression analysis

\begin{tabular}{lccc}
\hline Variable & $\begin{array}{l}\text { Partial regression } \\
\text { coefficient }\end{array}$ & $\begin{array}{l}\text { Partial correlation } \\
\text { coefficient }\end{array}$ & $F$ value \\
\hline Constant & 0.0333 & & 3.9134 \\
Age (years) & 0.0001 & 0.0218 & 0.1790 \\
Sex (1:male, 2:female) & -0.0044 & -0.0551 & 1.1518 \\
Duration of diabetes (years) & 0.0004 & 0.0903 & 3.1082 \\
HbA (\%) & -0.0014 & -0.0500 & 0.9474 \\
CMV-Hb (pmolCMV/mgHb) & -0.0003 & -0.0678 & 0.2839 \\
& & & 0.0787 \\
Stepwise forward selection analysis & & & 0.1876 \\
Constant & 0.0212 & & \\
Duration of diabetes (years) & 0.0004 & 0.1068 & 0.0009 \\
CMV-Hb(pmolCMV/mgHb) & -0.0003 & -0.0727 & 0.0367 \\
\end{tabular}

$H b A_{l c}$ stable fraction of glycohaemoglobin, $C M V$ - $H b$ haemoglobin with carbonyl methylated valine residue, a marker for advanced glycation endoproducts of haemoglobin [32]

there were no significant differences among the groups. The $m t D N A A 3243 G$ mutation rate in the healthy subjects is shown (Fig. 2). The value in the newborn group was negligible $(0.0003 \pm 0.0001 \%$ (mean \pm SEM), not detected in 83 out of 98 subjects); however, both the young group and the middle-aged group showed higher mutation rates $(0.0052 \pm 0.0003 \%$, not detected in 2 out of 93 young subjects, and $0.0043 \pm 0.0004 \%$, not detected in 7 out of 99 middleaged subjects, both $p<0.01$ vs the newborn group). 
The mutation rates of $m t D N A A 3243 G$ increased agedependently in the diabetic patients and the mutation was always detectable $(0.0115 \pm 0.0001 \%$ in the young group, $0.0158 \pm 0.0001 \%$ in the middle-aged group, $p<0.05$ vs the young group, and $0.0231 \pm 0.0040$ in the elderly group, $p<0.05$ vs both the young group and the middle-aged group, Fig. 3). There was no association between the somatic $A 3243 G$ mutation rate and the treatment of diabetes (diet only; $n=55$, \% mutation $=0.0233 \pm 0.0070$, hypoglycaemic reagents; $n=187$, $\%$ mutation $=0.0161 \pm 0.0010$, insulin therapy; $n=141$, $\%$ mutation $=0.0212 \pm 0.0045$ ) or BMI. A comparison between the age-matched healthy subjects and diabetic patients (41-60 years-old) is shown (Fig. 4). The mutation rate was about fourfold higher in the diabetic patients than in the healthy subjects $(p<0.01)$.

Multiple regression analysis of factors associated with the A3243G mutation. The correlation between the mutation rate of $A 3243 G$ in the diabetic patients and the clinical parameters is listed (Table 2 ). The mutation rate was not correlated with the age of the patients, but with the estimated duration of diabetes. The correlation was then analysed using the stepwise forward selection method as shown in Table 2 . Only the duration of diabetes, (and not age) was correlated with the rate of mutation of $m t D N A$ A3243G ( $p=0.0367)$. The rate of $m t D N A A 3243 G$ increased in relation to the estimated duration of diabetes and the mutation rate in the longest duration group ( $\geq 16$ years) was about twice than that in the shortest duration group $(\leq 5$ years, $p<0.05$, Fig. 5$)$.

\section{Discussion}

We first recognized accumulation of the somatic $m t D N A A 3243 G$ from healthy subjects after birth, a result that is consistent with a previous report [6]. The upper limit of measurable range for $A 3243 G$ was set at $0.1 \%$ in the assay system; thus, $A 3243 G$ carriers with heteroplasmy over $0.1 \%$ were excluded. Furthermore, no mutation was detected in umbilical venous blood cells. Taken together, these results suggest that $A 3243 G$ detected by our assay can be acquired as a somatic mutation. However, it is difficult to completely separate somatic mutation from clonal expansion of an inherited $m t D N A A 3243 G$; thus, we should investigate further the $A 3243 G$ mutation in umbilical blood cells from a large number of neonate subjects as well as in the buccal mucosa or hair follicles having fast cell cycles from adult subjects.

Our second finding was a marked increase of the mutation rate between the newborn and the young groups but not between the young and the middleaged groups. Preliminary evaluation in the non-diabetic subjects older than 90 years showed markedly higher rates than those in the young and middle-aged groups. Therefore, somatic mutation in healthy subjects could possibly increase dependent on age.

Our third finding was an age-dependent increase and a higher mutation rate in the diabetic subjects compared with the age-matched healthy subjects. Multiple regression analysis showed a significant correlation between detection of the $A 3243 G$ and the estimated duration of diabetes, but no correlation with age. In the preliminary experiments, we could not detect somatic mutations in human monocytic leukaemia-derived THP-1 cells cultured under a high glucose condition $(50 \mathrm{mmol} / \mathrm{l})$ for 3 months, which suggested that somatic mutation did not occur after such a short period or that the mutant $m t D N A$ was excluded during cell division. Therefore, considering the lifespan of peripheral blood cells, $m t D N A A 3243 G$ in peripheral blood cells could reflect accumulation of somatic mutations in bone marrow stem cells which persist in the peripheral blood cells. The carriers of $A 3243 G$ show an age-related decrease of heteroplasmy in their blood cells, because the tissues with a high replication rate tend to eliminate cells having a higher rate of mutated $m t D N A$ [22]. Although we did not assess the elimination of the mutation by cells with a very low rate of $A 3243 G(<0.1 \%)$, the diabetes duration-associated increase in the rate of $A 3243 G$ seen in our study could reflect a more potent effect of diabetes on the mutation, compared with its elimination by cell division.

It was reported that $84 \%$ of the subjects with impaired glucose tolerance and diabetes shared a 4977 base pair deletion of $m t D N A$, whereas only $41 \%$ of the age-matched healthy subjects had this deletion [23]. A recent report also showed that JCR:LA-corpulent rats characterized by insulin resistance, compensatory hyperinsulinaemia, hypertriglyceridaemia and atherosclerosis showed mtDNA deletion and mutation by ageing and hyperglycaemia [24]. These reports which evaluated somatic mutations in post mitotic tissues, suggest that diabetes can accelerate ageing-induced somatic mutation in mtDNA. Thus, it might be interesting to examine whether or not this common deletion is detectable in peripheral blood cells.

At present, there is no clinical marker that can be used to assess persistent hyperglycaemia for a longer period than $\mathrm{HbA}_{1 \mathrm{c}}$. Since the somatic $A 3243 G$ seems to show a diabetes duration-dependent increase, this could be a potent marker of hyperglycaemia. A persistent increase of ROS due to long-term hyperglycaemia or insulin resistance-induced compensatory hyperinsulinaemia might not only cause somatic mutation, but also the development of diabetic complications [25]. Thus, it is necessary to assess the role of long-term enhancement of ROS to predict diabetic complications. Although we did not assess the short-term markers of ROS such as 8-OH-2'-deoxyguanosine or 8 -iso-PGF2 $\alpha$, preliminarily evaluation in the subjects with severe retinopathy or nephropathy showed higher 
somatic $A 3243 G$ mutation than in the subjects without complications. Thus, somatic $A 3243 G$ could also be a useful marker of persistent oxidative stress and microvascular complications. In summary, to clarify the clinical meaning of the somatic $A 3243 G$, its association with glycaemic control, other markers of ROS, and diabetic complications should be investigated in a prospective study.

Of interest, mtDNA $A 3243 G$ itself increases the intra-cellular production of ROS [27], which could in turn cause secondary somatic mutations [28]. However, it is not clear if the degree of somatic A3243G seen in our study has a pathological effect or not. Oxidative stress-related DNA damage to the islets reduces the beta-cell mass [29]. If the somatic $A 3243 G$ accumulates in the islets, there is a possibility that it could become a factor in the progression of diabetes. Recently we found that the $A 3243 G$ mutation in the leucinespecific tRNA corresponding to the codon UUR $t R N A^{\text {Leu }}(U U R)$ induced a lack of modification of uridine at the first position of anticodon [30]. This suggests that such mutant $t R N A^{L e u}(U U R)$ can recognize all four nucleotides at the third position of the codon, giving rise to the translation of not only the usual UUR ( $\mathrm{R}=\mathrm{A}$ or $\mathrm{G}$ ) leucine codons but also UUY ( $\mathrm{Y}=\mathrm{C}$ or U) phenylalanine codons, which could eventually lead to the incorporation of leucine into phenylalanine sites at a certain rate [31]. The resulting synthesis of premature proteins due to this mistranslation could affect cells considerably, even if there is only a minor amount of the $A 3243 G$ mutant $m t D N A$.

In conclusion, we evaluated the somatic $m t D N A$ $A 3243 G$ in the peripheral blood cells in both healthy and diabetic subjects. The pathological significance of this mutation and its usefulness as a clinical marker should be investigated further.

\section{References}

1. Ouweland JMW van den, Lemkes HHP, Ruitenbeek W et al. (1992) Mutations in mitochondrial tRNALeu(UUR) gene in a large pedigree with maternally transmitted type II diabetes mellitus and deafness. Nat Genet 1:368-371

2. Kadowaki T, Kadowaki H, Mori Y et al. (1994) A subtype of diabetes mellitus associated with a mutation of mitochondrial DNA. N Eng J Med 330:962-968

3. Kobayashi Y, Momoi M-Y, Tominaga K et al. (1990) A point mutation in the mitochondrial tRNA(Leu)(UUR) gene in MELAS(mitochondrial myopathy, encephalopathy, lactic acidosis and stroke-like episodes). Biochem Biophys Res Commun 173:816-822

4. Goto Y, Nonaka I, Horai S (1990) A mutation in the tRNA(Leu)(UUR) gene associated with the MELAS subgroup of mitochondrial encephalopathies. Nature 348:651653

5. Kobayashi Y, Momoi MY, Tominaga K et al. (1991) Respiration-deficient cells are caused by a single point mutation in the mitochondrial tRNA-Leu(UUR) gene in mitochondrial myopathy, encephalopathy, lactic acidosis, and strokelike episodes (MELAS). Am J Hum Genet 49:590-599
6. Munscher C, Muller-Hocker J, Kadenbach B (1993) Human aging is associated with various point mutations in tRNA gene of mitochondrial DNA. Biol Chem 374:10991104

7. Corral-Debrinski M, Horton T, Lott MT, Shoffner JM, Beal MF, Wallace DC (1992) Mitochondrial DNA deletion in human brain: regional variability and increase with advanced age. Nat Genet 2:324-329

8. Ozawa T (1995) Mechanism of somatic mitochondrial DNA mutations associated with age and diseases. Biochim Biophys Acta 1271:177-189

9. Kadenbach B, Muncher C, Frank V, Muller-Hocker J, Napiwotzki J (1995) Human aging is associated with stochastic somatic mutations of mitochondrial DNA. Mutat Res 338:161-172

10. Michikawa Y, Mazzucchelli F, Bresolin N, Scarlato G, Attardi G (1999) Aging-dependent large accumulation of point mutations in the human mtDNA control region for replication. Science 286:774-779

11. Fliss MS, Usadel H, Caballero OL, Wu L, Buta MR, Eleff SM (2000) Facile detection of mitochondrial DNA mutations in tumors and bodily fluids. Science 287:20172019

12. Corral-Debrinski M, Shoffner JM, Lott MT, Wallace DC (1992) Association of mitochondrial DNA damage with aging and coronary atherosclerotic heart disease. Mutat Res 275:169-180

13. Corral-Debrinski M, Horton T, Lott MT et al. (1994) Marked changes in mitochondrial DNA deletion levels in Alzheimer brains. Genomics 23:471-476

14. Horton TM, Graham BH, Corral-Debrinski M et al. (1995) Marked increase in mitochondrial DNA deletion levels in the cerebral cortex of Huntington's disease patients. Neurology 45:1879-1883

15. Hayashi J, Ohta S, Kagawa Y et al. (1994) Nuclear but not mitochondrial genome involvement in human age-related mitochondrial dysfunction. Functional integrity of mitochondrial DNA from aged subjects. J Biol Chem 269:6878-6883

16. Williamson JR, Chang K, Frangos M et al. (1993) Hyperglycemic pseudohypoxia and diabetic complications. Diabetes 42:801-813

17. Hunt JV, Dean RT, Wolff SP (1988) Hydroxyl radical production and autoxidative glycosylation. Glucose autoxidation as the cause of protein damage in the experimental glycation model of diabetes mellitus and aging. Biochem $\mathrm{J}$ 256:205-212

18. Yan H, Harding JJ (1997) Glycation-induced inactivation and loss of antigenicity of catalase and superoxide dismutase. Biochem J 328:599-605

19. Kashiwagi A, Shinozaki K, Nishio Y et al. (1999) Endothelium-specific activation of $\mathrm{NAD}(\mathrm{P}) \mathrm{H}$ oxidase in aortas of exogenously hyperinsulinemic rats. Am J Physiol 277:E976-E978

20. Dandona P, Thusu K, Cook S et al. (1996) Oxidative damage to DNA in diabetes mellitus. Lancet 347:444-445

21. Hinokio Y, Suzuki S, Hirai M, Chiba M, Hirai A, Toyota T (1999) Oxidative damage in diabetes mellitus: its association with diabetic complications. Diabetologia 42:995-998

22. 't Hart LM, Jansen JJ, Lemkes HH, Knijff P de, Maassen JA (1996) Heteroplasmy levels of a mitochondrial gene mutation associated with diabetes mellitus decrease in leucocyte D upon aging. Hum Mutat 7:193-197

23. Liang P, Huges V, Fukagawa NK (1997) Increased prevalence of mitochondrial DNA deletions in skeletal muscle of older individuals with impaired glucose tolerance: possible marker of glycemic stress. Diabetes 46:920-923 
24. Fukagawa NK, Li M, Liang P, Russell JC, Sobel BE, Absher PM (1999) Aging and high concentrations of glucose potentiate injury to mitochondrial DNA. Free Radic Biol Med 27:1437-1443

25. Suzuki S, Hinokio Y, Komatu K et al. (1999) Oxidative damage to mitochondrial DNA and its relationship to diabetic complications. Diabetes Res Clin Pract 45:161-168

26. Marfella R, Quagliaro L, Nappo F, Ceriello A, Giugliano D (2001) Acute hyperglycemia induced an oxidative stress in healthy subjects. J Clin Invest 108:635-636

27. Zhang J, Yoneda M, Naruse K et al. (1998) Peroxide production and apoptosis in cultured cells carrying mtDNA mutation causing encephalomyopathy. Biochem Mol Biol Int 46:71-79

28. Kovalenko SA, Tanaka M, Yoneda M, Iakovlev AF, Ozawa T (1996) Accumulation of somatic nucleotide substitutions in mitochondrial DNA associated with the 3243 A-to-G tRNA(leu)(UUR) mutation in encephalomyopathy and cardiomyopathy. Biochem Biophys Res Commun 222:201-207

29. Sakuraba H, Mizukami H, Yagihashi N, Wada R, Hanyu C, Yagihashi S (2002) Reduced beta-cell mass and expression of oxidative stress- related DNA damage in the islet of Japanese Type II diabetic patients. Diabetologia 45:85-96

30. Yasukawa T, Suzuki T, Ueda T, Ohta S, Watanabe K (2000) Modification defect at anticodon wobble nucleotide of mitochondrial tRNAs(Leu)(UUR) with pathogenic mutations of mitochondrial myopathy, encephalopathy, lactic acidosis, and stroke-like episodes. J Biol Chem 275:4251-4257

31. Yasukawa T, Suzuki T, Ishii N, Ohta S, Watanabe K (2001) Wobble modification defect in tRNA disturbs codon-anticodon interaction in a mitochondrial disease. EMBO J 20:4794-4802

32. Uchimura T, Nakano K, Hashiguchi T et al. (2001) Elevation of $\mathrm{N}$-(Carboxymethyl)valine residue in hemoglobin of diabetic patients. Diabetes Care 24:891-896 